CARDIOVASCULAR MEDICINE

\title{
The safety and effectiveness of a nurse led cardioversion service under sedation
}

\author{
L Boodhoo, G Bordoli, A R Mitchell, G Lloyd, N Sulke, N Patel
}

Heart 2004;90:1443-1446. doi: 10.1136/hrt.2004.034900

See end of article for authors' affiliations

.....................

Correspondence to: Dr Lana Boodhoo, Department of Cardiology, Eastbourne General Hospital, Eastbourne BN21 2UD, UK; lboodhoo@aol.com

Accepted 22 April 2004
Objective: To assess the safety and effectiveness of nurse led elective cardioversion of atrial fibrillation under sedation.

Design: Prospective, longitudinal study.

Setting: Cardiac catheterisation laboratory and recovery area of a district general hospital.

Patients: 300 patients referred for elective cardioversion of persistent atrial fibrillation.

Interventions: Pre-procedure evaluations (history, physical examination, blood tests), consent, sedation administration, cardioversions, and post-procedure monitoring until discharge by advanced life support certified coronary care unit nurses trained in the techniques. A doctor was immediately available if required but not present.

Main outcome measures: Success rates at discharge and at six weeks, energy delivered, number of shocks, dose of sedation, immediate, 24 , and 48 hour patient perceptions, complications, waiting times, and cost effectiveness.

Results: Cardioversion success rate was $87 \%$ at discharge and $48 \%$ at six weeks. Mean (SD) cumulative energy was 497 (282) J and number of shocks 1.6 (0.8). Mean (SD) dose of sedation was 23 (9) mg intravenous diazepam. No patient required reversal of sedation, airway support, or medical intervention. Ninety eight per cent of patients had no pain or recall of the procedure. Four patients who were adequately anticoagulated experienced embolic phenomena. Ninety eight per cent of patients would repeat the procedure if necessary. Without requirement for a physician or anaesthetist, waiting times for elective cardioversion fell from three months to under four weeks. There was a significant reduction in the estimated cost of the procedure from $£ 337$ with general anaesthesia to $£ 130$ with nurse led sedation and cardioversion ( $p<0.001)$.

Conclusion: With appropriate training, a nurse led cardioversion service with sedation is safe, effective, well tolerated, and cost efficient.
D irect current cardioversion remains the most effective method for restoring sinus rhythm in patients with persistent atrial fibrillation (AF). ${ }^{1}$ Cardioversion is traditionally performed under short acting general anaesthesia but several studies have documented that it may be performed as safely, effectively, and more economically under sedation administered by non-anaesthetist physicians. $^{2-5}$ Another category of staff possessing the technical and cognitive skills required for the performance of elective cardioversion and administration of sedation is advanced cardiac life support (ALS) certified coronary care unit nurses. ${ }^{6}$

There has been considerable support among nurses for role expansion in the critical care setting. ${ }^{7}$ This, combined with a simultaneous reduction of junior doctors' working hours in the UK and the need for cost containment within the National Health Service (NHS), prompted the development of a local initiative to extend nursing practice to include elective cardioversion of patients with persistent AF. ${ }^{8}$

\section{Study aims}

The primary aim of this study was to prospectively determine the short and medium term safety and effectiveness of a nurse led cardioversion service. Secondary outcomes were cost analysis of the procedure and patient acceptability.

\section{PATIENTS AND METHODS}

All patients referred for elective cardioversion of haemodynamically stable persistent AF were prospectively assessed in this single centre study between July 2002 and July 2003. The study was performed in accordance with the Declaration of
Helsinki and the protocol was approved by the local research and ethics committee. Written informed consent was obtained from all patients. Inclusion criteria were AF of greater than 24 hours' duration that required external cardioversion and no reversible cause of AF. At least four weeks of anticoagulation with warfarin with an international normalised ratio (INR) $>2.0$ was required. Patients were excluded for the following reasons: serum potassium $<3.5 \mathrm{mmol} / \mathrm{l}$, INR $<2.0$, known allergy to any drug normally used in the procedure, known sedation related complication, clinical evidence of digitalis toxicity, obstructive sleep apnoea, or resting oxygen saturation measured by oximetry of less than $90 \%$. Patients presented for preprocedure blood tests and a 12 lead ECG 48 hours before the procedure. They were given an information sheet about the procedure that provided aftercare instructions and a telephone contact number for the coronary care unit.

All nurses taking part in the study had at least one year of coronary care unit experience, were ALS certified, had attended a course in safe sedation practice, and had performed a minimum of 20 supervised sedation-cardioversion procedures before study commencement. The nurse led cardioversion protocol required the nurses to evaluate patients before the procedure on the day of cardioversion. This entailed taking information on a history of medical

Abbreviations: $\mathrm{AF}$, atrial fibrillation; $\mathrm{ALS}$, advanced cardiac life support; INR, international normalised ratio; UKCC, United Kingdom Central Council 
illness or conditions that can alter the patients' response to sedation, a history of prior adverse reactions to general or local anaesthesia and sedation, current medication, allergies, and any history of alcohol or drug abuse. Nurses also performed a focused physical examination of vital signs, auscultation of the heart and lungs, and airway evaluation. Nurses reviewed the patients' pre-procedure laboratory tests to ensure INRs were between 2.0-3.0 and that potassium concentrations were adequate $(\geqslant 3.5 \mathrm{mmol} / \mathrm{l})$. Nurses counselled patients, obtained consent for the procedure, and performed intravenous cannulation.

All cardioversion procedures took place in the cardiac catheterisation laboratory that was equipped with monitoring and resuscitation equipment and was adjacent to an eight bed dedicated recovery area also equipped with non-invasive monitors. Three nurses were required, two to carry out sedation and cardioversion in the procedure room and one to staff the recovery area. A cardiology registrar was immediately available if required but was not present. One of the two nurses in the procedure room had the designated role of monitoring patient safety, keeping records, and administering sedation while the other nurse performed the cardioversion.

Patients were monitored by continuous pulse oximetry, ECG, observation or auscultation to assess respiratory function, and pulse and blood pressure measurement at five minute intervals. These were recorded before, during, and after administration of sedation, during and upon completion of the procedure, during recovery, and at the time of discharge. Supplemental oxygen was given through a facemask before and after the procedure. Intravenous diazepam was given for sedation as Diazemuls (Dumex, Exeter, UK) with an initial bolus of $10 \mathrm{mg}$, then smaller increments of $2.5 \mathrm{mg}$ every minute until a satisfactory depth of sedation was achieved or until a maximum of $40 \mathrm{mg}$ was administered. Sedation was given until the central nervous system was depressed, with the patient being able to respond purposefully to tactile or painful stimuli and maintaining an independent airway, adequate spontaneous ventilation, and cardiovascular function. Coadministration of narcotic agents was prohibited.

All cardioversions were undertaken with a single defibrillator (Physio-Control Lifepak 9 defibrillator, Physio-Control Corp, Redmond, Washington, USA) that used damped monophasic shock waveforms. Gel pads were applied to the patient and the nurse performing the cardioversion procedure applied synchronised shocks of 200 or $360 \mathrm{~J}$ through paddles with pressure in the anterolateral (right infraclavicularventricular apex) or anteroposterior (body of sternuminterscapular) position with a maximum of three shocks administered in each procedure. After the cardioversion, patients were placed in the recovery position and vital signs were monitored every five minutes for the first 30 minutes, then every 15 minutes until the patient was fit for discharge

\begin{tabular}{|ll|}
\hline Table 1 Patient characteristics $(\mathrm{n}=300)$ & \\
\hline Number of procedures & 394 \\
Women & $39 \%$ \\
Age (years) & $71(10)$ \\
Height $(\mathrm{cm})$ & $173(11)$ \\
Weight $(\mathrm{kg})$ & $79(17)$ \\
Body mass index $\left(\mathrm{kg} / \mathrm{m}^{2}\right)$ & $26(4)$ \\
Left atrial diameter $(\mathrm{cm})$ & $4.6(0.8)$ \\
Ejection fraction $(\%)$ & 65 \\
Alcohol intake $(u n i t s /$ week) & 3 \\
Duration of atrial fibrillation $>6$ months & $72 \%$ \\
\hline Values are expressed as mean (SD). & \\
\hline
\end{tabular}

(a minimum of two hours after the procedure). For discharge, the patient was required to be alert and oriented with stable vital signs that had returned to baseline and to be accompanied by a responsible adult to take them home and to report any post-procedure complications. Patients were advised not to drive or drink alcohol for 48 hours after the procedure.

Cardioversion was considered successful if sinus rhythm was achieved and maintained to the time of discharge. Patient perception of the procedure was assessed before discharge and at 24 hours and 48 hours after the procedure by custom designed questionnaires. These evaluated shock recall, side effects, and willingness to repeat the procedure under sedation if medically indicated. Post-procedure questionnaires were returned in prepaid envelopes. Patients were clinically reviewed by a physician six weeks after cardioversion with a 12 lead ECG.

The cost of the procedure was estimated from local NHS costs of drugs and consumables used and nursing staff salaries.

\section{RESULTS}

Three hundred and ninety four elective procedures were performed on 300 patients (61\% men, mean (SD) age 71 (10) years) with persistent AF during the study period, compared with 306 physician led procedures performed during the corresponding period during the previous year. Tables 1 and 2 describe the clinical characteristics of the patients. All patients consented to nurse led cardioversion.

\section{Effectiveness}

The mean intravenous dose of diazepam was 23 (9) $\mathrm{mg}$. The mean number of shocks was $1.6(0.8)$ and cumulative energy delivered was 497 (282) J. The cardioversion success rate at discharge was $87 \%$ and $48 \%$ of patients were in sinus rhythm at the six week review.

\section{Safety}

No patient experienced respiratory depression or required reversal of sedation, ventilation, or anaesthetist or physician intervention. Five patients experienced transient hypotension after the procedure that resolved with intravenous fluids. The mean dose of Diazemuls administered to these patients was 24 (5) mg. All patients were discharged on the same day as their procedure. Six procedures were abandoned because the patients could not be sedated adequately after administration of a maximum of $40 \mathrm{mg}$ Diazemuls. These patients were noted to have recognised adverse clinical characteristicseither obesity, with body mass indices exceeding $35 \mathrm{~kg} / \mathrm{m}^{2}$ (three patients), or habitual use of benzodiazepines for insomnia (three patients). There were no immediate or six

\begin{tabular}{|ll|}
\hline $\begin{array}{l}\text { Table } 2 \text { Underlying aetiology and } \\
\text { antiarrhythmic drug treatment }\end{array}$ \\
\hline $\begin{array}{l}\text { Underlying aetiology } \\
\text { Valvar }\end{array}$ \\
Ischaemic & $4 \%$ \\
Congestive cardiac failure & $10 \%$ \\
Hypertension & $4 \%$ \\
Pulmonary & $50 \%$ \\
Unknown & $4 \%$ \\
Antiarrhythmics & $28 \%$ \\
Digoxin & \\
Class I & $29 \%$ \\
Class II & $3 \%$ \\
Class III & $15 \%$ \\
Class IV & $38 \%$ \\
\hline & $2 \%$ \\
\hline
\end{tabular}


week deaths as a result of the procedure. Four patients (1\%), despite adequate anticoagulation with warfarin, experienced systemic emboli up to four days after the procedure: three were cerebrovascular events and one a splenic infarct. There were no other major complications.

\section{Tolerability}

Questionnaires were completed by $90 \%$ of patients. Ninety eight per cent of these had total amnesia of the procedure. Forty per cent of patients reported minor symptoms of dermal injury (redness, itching, or soreness) at the sites of paddle application 24 hours after the procedure. By 48 hours after the procedure the proportion reporting these symptoms had decreased to $21 \%$. Thirty three per cent experienced minor sedative side effects (drowsiness, unsteadiness) at 24 hours. At 48 hours after the procedure $17 \%$ had residual symptoms. Ninety five per cent of patients said they would repeat the procedure if medically indicated, and $98 \%$ would have the cardioversion performed under sedation.

\section{Cost effectiveness and waiting times}

The cost of the cardioversion under general anaesthesia was estimated to be $£ 337$ for each procedure, compared with $£ 130$ for each nurse led cardioversion, a $61 \%$ reduction. With an annual number of elective cardioversion procedures of 400 , this equates to cost savings of $£ 82800$. After one year of nurse led cardioversion the waiting time for elective cardioversion fell from three months to under four weeks.

\section{DISCUSSION}

This study has shown that nurse led elective cardioversion under sedation is safe, effective, well tolerated, and cost efficient. Although defibrillation and cardioversion have been traditionally regarded as skills performed exclusively by medical staff, it has become routine practice for nurses to defibrillate and initiate resuscitative measures during cardiac arrest. Many nurses have thus already gained competence in this technique. ALS trained coronary care nurses possess the technical skills required for performance of cardioversion, in addition to the core skills required for administration of sedation. ${ }^{6}$ The American College of Physicians/American College of Cardiology/American Heart Association Task Force on Cardiology of the American College of Physicians' Clinical Privileges Project recommends participation in a minimum of eight supervised cardioversions to achieve competence in the necessary cognitive and technical skills. ${ }^{6}$ It also recommends that a minimum of four procedures a year be required to maintain competence after initial certification. These targets have already been met and exceeded by most coronary care unit nurses at Eastbourne Hospital.

\section{Safety}

There is concern that sedation with benzodiazepines may result in airway dysfunction requiring the intervention of anaesthetic personnel, and that the inability of pulse oximetry to detect hypoventilation and carbon dioxide retention can lead to arrhythmias and deepening coma if hypercapnia is undetected and left untreated. ${ }^{10}$ Although these concerns are valid, in this series of 394 procedures no such complication occurred. This may have been due to careful patient selection to exclude those considered at high risk and to the use of intravenous Diazemuls as the sedative agent. Several published studies have confirmed the safety and efficacy of this agent for inducing adequate levels of sedation for cardioversion. ${ }^{11-13}$ In two recent studies in which physicians administered diazepam for cardioversion in the absence of an anaesthetist the mean doses used were $27.2 \mathrm{mg}$ and $28.1 \mathrm{mg}$, with diazepam alone providing adequate sedation in $97 \%$ patients with no complications. ${ }^{35}$ In contrast, the mean dose of diazepam administered by nurses was $23.2 \mathrm{mg}$, a 15-19\% reduction in the doses administered by doctors, with similar satisfactory levels of sedation achieved. This discrepancy may be explained by differences in the depth of sedation incurred with these doses. In the previous studies of physician led cardioversion, sedation was given with the loss of eyelash reflex as an end point for satisfactory sedation. The doses that nurses used in the present study incurred relatively lighter levels of central nervous system depression sufficient to abolish recall, but preserving airway patency, spontaneous ventilation, cardiovascular function, and protective reflexes. This is highly advantageous in reducing the potential for airway problems and hypotension, facilitating earlier patient ambulation and causing fewer sedative side effects. The increased sensitivity of the elderly to sedative hypnotics highlights the desirability of careful dose titration to prevent recall while maintaining protective reflexes. ${ }^{14}$ Coronary care nurses performing the procedure were required to be ALS certified, therefore having appropriate basic training in airway management, and to have attended a formal course on safe sedation practice.

\section{Tolerability}

The proportion of patients experiencing skin burns is comparable with previous series of patients undergoing cardioversion. ${ }^{15}$ The incidence of sedative side effects experienced 24 and 48 hours after the procedure, generally described as mild, probably reflects the age related impaired clearance of the drug. ${ }^{14}$ There was a high degree of patient satisfaction and willingness to repeat the procedure if indicated.

\section{Cost effectiveness and number of procedures}

Substitution of ALS trained coronary care nurses for physicians and anaesthetists resulted in similar clinical outcomes but with important cost savings. Another advantage was an increased number of procedures over the previous year when the service was physician led. Eliminating the limitations of having to coordinate the procedure at a mutually acceptable time for cardiology staff and physician allowed for fewer delays and more procedures to be accomplished, and this translated into shorter waiting times. This is potentially beneficial in terms of clinical outcomes and the likelihood of maintaining sinus rhythm after cardioversion. ${ }^{16}$

\section{Medicolegal considerations}

There is understandable concern about the potential legal implications of the practice of nurse led sedation and cardioversion and the need for full medicolegal coverage in the event of complications. Medicolegally a nurse may administer sedation with the appropriate supervision, training, certification, and support of the hospital. Doctors who delegate medical duties to nurses must be satisfied that the nurses are competent to carry them out and should retain ultimate responsibility for the management of these patients. ${ }^{17}$ Training and supervision are thus essential until proficiency is satisfactory. The nurse should be responsible for obtaining consent for the procedure and for discussing the outcome with the patient after the procedure; however, further patient management should remain the responsibility of the doctor. Nurses must acknowledge any limits in their competence and decline duties unless they can perform them safely and skilfully. In the UK, nurses remain subject to United Kingdom Central Council (UKCC) regulations and are accountable to the council. Nursing managers must ensure that local policies are based on UKCC principles and that nurses are assisted in suitably adjusting their practice. $^{18}$ Patients must be informed adequately about the nature of 
the post holder's profession, role, training, and experience. The new responsibilities must be formally acknowledged by the hospital, and relevant insurers and job descriptions must be updated as necessary. Nurses are covered by the NHS Hospital and Community Health Services indemnity against claims of medical negligence.

\section{Recommendations}

For nurses to undertake expanded roles such as those discussed in this study, there is a need for appropriate education and training as well as assessment strategies to ensure that nurses are and remain competent in the performance of these skills. Thorough and appropriate preprocedure evaluation increases the likelihood of satisfactory sedation, decreases the likelihood of adverse outcomes, and requires good physical assessment skills. Formal education and training in the pharmacology of agents commonly used during sedation, in the role of antagonists, and in airway management will also improve the likelihood of satisfactory sedation and reduce the risk of adverse outcomes. Nurses administering sedation must have the skills to rescue patients with complications (tracheal intubation and use of antagonists). Regular audit and continuing education with opportunities to attend relevant courses are also necessary.

\section{Conclusions}

A nurse led cardioversion service is safe, effective, and well tolerated and it results in significant cost savings. Medicolegally a nurse may administer sedation with the appropriate supervision, training, certification, and support of the hospital. Careful patient selection is essential to exclude high risk patients. Training should include attendance at a recognised teaching course in safe sedation practice, which should address the pharmacology of the drugs and their antagonists and airways management. A designated appropriately trained supervising physician should be immediately available during the procedure. Nurses should be regularly audited, take part in continuing education, and have opportunities to attend relevant courses.
Authors' affiliations

L Boodhoo, G Bordoli, A R Mitchell, G Lloyd, N Sulke, N Patel, Department of Cardiology, Eastbourne General Hospital, Eastbourne, UK

\section{REFERENCES}

1 Levy S, Breithardt G, Campbell RW, et al. Atrial fibrillation: current knowledge and recommendations for management. Working group on arrhythmias of the European Society of Cardiology. Eur Heart J 1998;19:1294-320.

2 Raipancholia R, Sentinella L, Lynch M. Role of conscious sedation for external cardioversion. Heart 2001:86:571-2.

3 Pugh PJ, Spurrell P, Kamalvand K, et al. Sedation by physician with diazepam for DC cardioversion of atrial arrhythmias. Heart 2001;86:572-3.

4 Goldner BG, Baker J, Accordino A, et al. Electrical cardioversion of atrial fibrillation or flutter with conscious sedation in the age of cost containment. Am Heart J 1998;136:961-4.

5 Mitchell AR, Chalil S, Boodhoo L, et al. Diazepam or midazolam for external DC cardioversion (the DORM study). Europace 2003;5:391-5.

6 Yurchak PM, Williams SV, Achord JL, et al. Clinical competence in elective direct current (DC) cardioversion: a statement for physicians from the ACP/ ACC/AHA task force on clinical privileges in cardiology. J Am Coll Cardiol 1993;22:336-9.

7 Hind $M$, Jackson D, Andrewes $C$, et al. Exploring the expanded role of nurses in critical care. Intensive Crit Care Nurs 1999;15:147-53

8 Department of Health. NHS Management Executive. Junior doctors: the new deal. London: Department of Health, 1991.

9 Gross J, Bailey P, Connis R, et al. Practice guidelines for sedation and analgesia by non-anesthesiologists. Anaesthesiology 2002;96:1004-17.

10 Harrison SJ, Mayet J. Physician administered sedation for DC cardioversion. Heart 2002;88:117-8.

11 Muenster JJ, Rosenberg MS, Carleton RA, et al. Comparison between diazepam and sodium thiopental during DC countershock. JAMA 1967; 199:758-60.

12 Forssell G, Nordlander R, Nyquist O, et al. Diazepam in cardioversion. Acta Med Scand 1975;197:255-6.

13 Woodbridge JH. Experiences with electrical cardioversion in an outpatient clinic. J Miss State Med Assoc 1969;10:542-4.

14 Greenblatt DJ, Harmatz JS, Shader RI. Clinical pharmacokinetics of anxiolytics and hypnotics in the elderly: therapeutic considerations (part I). Clin Pharmacokinet 1991;21:165-77.

15 McNaughton GW, Wyatt JP, Byrne JC. Defibrillation: a burning issue in coronary care units! Scott Med J 1996;41:47-8.

16 Wijffels MC, Kirchhof CJ, Dorland R, et al. Atrial fibrillation begets atrial fibrillation: a study in awake chronically instrumented goats. Circulation 1995;92:1954-68.

17 General Medical Council. Professional conduct and discipline: fitness to practice. London: GMCl, 1993.

18 United Kingdom Central Council. The scope of professional practice. London: UKCC, 1992. 